\title{
ESTIMATES OF HARMONIC MEASURES
}

\author{
LENNART CARLESON
}

1. The theory of analytic functions of one complex variable can be based on essentially three different principles: (1) the Cauchy integral and the related power series expansions, (2) the geometric idea of conformal mapping and (3) the use of harmonic functions and the study of $\log |f(z)|$. In the development during the last fifty years the last aspect has been predominant and the work of R. Nevanlinna together with that of many other Scandinavian mathematicians has been of fundamental importance. To combine the aspects (2) and (3) one must be able to handle harmonic functions in terms of geometric conditions and this is how harmonic measures enter. I shall here give a short summary of the most important methods and mention some open problems. During the last decades the first method has been revived through the $\bar{\partial}$-equation and we now have methods where all three aspects can be made to work together.

Assume that $\mathscr{D}$ is a domain in the plane. We divide its boundary into two subsets, $\partial \mathscr{D}=E \cup \Gamma$, and we are interested in estimating the harmonic function in $\mathscr{D}$, $\omega(z ; E ; \mathscr{D})$ which $=1$ on $E$ and $=0$ on $\Gamma$, at some fixed interior point $z_{0}$ of $\mathscr{D}$. This point $z_{0}$ is always assumed not to be close to $\partial \mathscr{D}$. I shall schematically classify the situation in the following sections.

\section{2. $\mathscr{D}$ simply connected; $E$ an arc}

This case is the most well-known and there are three essentially equivalent methods

(A) the Ahlfors distortion theorem [1]

(B) Beurling's method of extremal length

(C) Carleman's differential inequality [4].

Since Beurling's method is the least known and the most flexible, I shall indicate the result.

We assume that $\beta$ is some fixed arc "close" to $z_{0}$ and that the geometry of $\mathscr{D}$ is well-behaved in this part of $\mathscr{D}$. Consider the family of all curves $\{\gamma\}$ joining $\beta$ 
to $E$ in $\mathscr{D}$ and all well-behaved non-negative functions $\varrho$ in $\mathscr{D}$ such that

We define

$$
\int_{\gamma} \varrho(z)|d z| \geqq 1, \quad \gamma \in\{\gamma\} .
$$

$$
\lambda(E ; \beta)^{-2}=\inf _{\varrho} \int_{\mathscr{Q}} \varrho(z)^{2} d x d y .
$$

Then

$$
\omega\left(z_{0} ; E ; \mathscr{D}\right) \asymp \exp \left\{-\pi \lambda^{2}\right\}
$$

where $\asymp$ indicates that the two sides are equal disregarding constants which can be found in terms of the local geometry around $z_{0}$.

Upper estimates of $\omega$ are obtained using particular choices of $\varrho$. In the case of a domain where segments $\theta_{x}, x_{0}<x<x_{1}$, parallel to the $y$-axis separate $z_{0}$ from $E$, we can choose

$$
\varrho(x, y)= \begin{cases}\frac{1}{\left|\theta_{x}\right|}\left(\int_{x_{0}}^{x_{1}} \frac{d t}{\left|\theta_{t}\right|}\right)^{-1}, & \begin{array}{l}
x_{0}<x<x_{1},(x, y) \in \theta_{x} \\
0
\end{array} \\
\text { otherwise }\end{cases}
$$

and obtain Ahlfors' distortion theorem.

To get lower estimates we observe that $\lambda(\beta, E) \cdot \lambda(\delta, \sigma)=1$, if $\delta$ and $\sigma$ are the arcs $\partial \mathscr{D} \backslash(\beta \cup E)$ and estimate $\lambda(\delta, \sigma)$ from above by special choices of $\varrho$.

\section{3. $E$ isolated from $\Gamma=\partial \mathscr{D}-E$}

We assume that $\Gamma$ contains some well-behaved part. A typical case is when $E$ is located inside a curve $\Gamma$.

We assume first that the complement of $E$ has a Green's function

$$
g(z)=\int_{E} \log |z-t| d \mu_{0}(t)+V .
$$

$C(E)=V^{-1}$ is the capacity of $E$ (observe that there are other normalizations). Then

$$
\omega\left(z_{0} ; E ; \mathscr{D}\right) \asymp C(E)
$$

with the same conventions as before. Since

$$
V=\inf _{\mu} \int_{E E} \log \frac{1}{|z-\zeta|} d \mu(z) d \mu(\zeta)
$$

for all $\mu \geqq 0, \mu(E)=1$, we have good possibilities to make geometric estimates. - If $g(z)$ does not exist $C(E)=\omega\left(z_{0} ; E ; \mathscr{D}\right)=0$.

In Nevanlinna's book [9] all this is set down in detail. 


\section{4. $\Gamma$ a smooth curve, $E$ close to $\Gamma$}

This is a very important case for studies of entire functions and behavior of analytic functions close to the boundary. The first results are by Nevanlinna [7] and Beurling [3] and the very useful lemma of Hall [6]. A rather complete solution can be formulated as follows. Even if the result has not been recorded, this type of estimate was known to Beurling and the methods are standard in $H^{p}$-theory. Nevanlinna's estimate [7] was based on the same idea.

To simplify the presentation we consider the upper half-plane and assume that $E \subset(0,1) \times(0,1 / 2)$ and choose $z_{0}=i$. Denote by $Q$ dyadic cubes obtained from $Q_{0}=(0,1) \times(0,1)$ and having one side on $y=0$. (F) is the family of positive measures on $Q_{0}$ which satisfy

(A) $\mu(Q) \leqq s(Q)$ for all such $Q$,

where $s(Q)$ is the side-length of $Q$. Let us furthermore denote by $Q^{\prime}$ the upper half of $Q$ and set $E\left(Q^{\prime}\right)=E \cap Q^{\prime}$. We then also assume

(B) $\mu\left(Q^{\prime}\right) \leqq \frac{C\left(E\left(Q^{\prime}\right)\right)}{C\left(E\left(Q^{\prime}\right)\right)+\left(1-C\left(E\left(Q^{\prime}\right)\right) / C\left(Q^{\prime}\right)\right)} \cdot s(Q)$.

We then have for $\mathscr{D}=$ upper half plane $\mathscr{H} \backslash E$

$$
\omega(i ; E ; \mathscr{D}) \asymp \sup _{(A),(B)} \mu(\mathscr{H}) .
$$

Let us first observe that the Green's function for $y>0$ with pole at $\zeta=\xi+i \eta$ satisfies

$$
G(z ; \zeta) \asymp \begin{cases}\log \frac{\eta}{|z-\zeta|}+O(1), & |z-\zeta| \leqq \frac{\eta}{2} \\ \frac{y \eta}{|z-\zeta|^{2}}, & |z-\zeta| \geqq \frac{\eta}{2} .\end{cases}
$$

We shall in the sequel relate measures $\mu$ and $v$ to each other by the relation

$$
d v(\zeta)=d \mu(\zeta) \cdot \eta^{-1}
$$

We then have the estimate for $z \in Q^{\prime}$

$$
u_{v}(z)=\int G(z ; \zeta) d v(\zeta) \geqq \text { Const. } \frac{\mu(Q)}{s(Q)} .
$$

Conversely, if $z \in Q$ and $\mu$ satisfies (A), then

$$
\begin{gathered}
\int_{|z-\zeta| \geqq s(Q) / 4} G(z ; \zeta) d v(\zeta) \leqq \text { Const. } y \int_{|z-\zeta| \geqq s(Q) / 4} \frac{d \mu(\zeta)}{|z-\zeta|^{2}} \\
\leqq \text { Const. } y \int_{s / 4}^{\infty} \frac{t d t}{t^{3}} \leqq \text { Const. }
\end{gathered}
$$


Let us assume that $E$ is located in $y \geqq 2^{-k-1}$. We first consider squares $Q$ of side $2^{-k}$. If $C\left(E\left(Q^{\prime}\right)\right)>0$, let $v_{Q}^{*}$ be the distribution of mass $C\left(E\left(Q^{\prime}\right)\right)$ on $E\left(Q^{\prime}\right)$ so that

$$
\int_{E\left(Q^{\prime}\right)} \log \frac{1}{|z-\zeta|} d v_{Q}^{*}(\zeta)=1, \quad z \in E\left(Q^{\prime}\right)
$$

An easy computation shows that on $E\left(Q^{\prime}\right)$

$$
\int G(z ; \zeta) d v_{Q}^{*}(\zeta) \asymp 1-\frac{C\left(E\left(Q^{\prime}\right)\right)}{C\left(Q^{\prime}\right)}+C\left(E\left(Q^{\prime}\right)\right) .
$$

Hence the maximal total mass that we can distribute on $E\left(Q^{\prime}\right)$ with a Green's potential $\leqq 1$ is

$$
\asymp \frac{C\left(E\left(Q^{\prime}\right)\right)}{C\left(E\left(Q^{\prime}\right)\right)+\left(1-C\left(E\left(Q^{\prime}\right)\right) / C\left(Q^{\prime}\right)\right)} .
$$

We define $v_{Q}=$ Const. $v_{Q}^{*}$ so that $\sup _{E\left(Q^{\prime}\right)} u_{v_{Q}}=1$. We do this for every $Q$ of side $2^{-k}$ and define $v^{(1)}=\sum v_{Q}$ for these $Q$ 's. From (4.3) follows that $u_{v}(z) \leqq$ Const.

Having defined $v^{(j)}$ and $\mu^{(j)}$ for squares of sides $2^{-j}, j \geqq m+1$, we consider $E\left(Q^{\prime}\right)$ for a square of side $2^{-m}$.

If

$$
u_{v^{(m+1)}}(z) \geqq 1 \quad \text { on } \quad E\left(Q^{\prime}\right)
$$

we put $v^{(m)}=\mu^{(m)}=0$ on $E\left(Q^{\prime}\right)$. If not, we define

$$
v^{(m)}(\zeta)=\text { Const. } v_{Q}(\zeta) \quad \text { on } \quad E\left(Q^{\prime}\right)
$$

where the constant $c$ is chosen so that $\inf _{E\left(Q^{\prime}\right)} u_{v(m+1)+c v_{Q}}(z)=1$. We go through the squares of size $2^{-m}$ and have defined $v^{(m)}$ by (4.4). We set $v=v^{(1)}$ and $\mu=\mu^{(1)}$.

It is clear from the construction that $u_{\nu} \geqq 1$ on $E$. Since $u_{v_{Q}} \asymp 1$ on $E\left(Q^{\prime}\right)$ it is easy to see that $u_{v^{(m+1)}}\left(z_{1}\right) / u_{v^{(m+1)}}\left(z_{2}\right) \asymp 1$ for $z_{1}, z_{2}$ on $Q^{\prime}$ for a square $Q$ of size $2^{-m}$ (this is essentially Harnack's inequality). It then follows from the construction that

and by (4.2)

We conclude

$$
u_{v}(z) \leqq \text { Const., for all } z \text {, }
$$

and

$$
\mu(Q) \leqq \text { Const. } s(Q), \text { for all } Q \text {. }
$$

$$
\omega(z) \asymp u_{v}(z)
$$

$$
\omega(i) \asymp u_{v}(i) \asymp \int \eta d v(\eta)=\mu(\mathscr{H}) .
$$

Conversely, let $\mu_{0}$ satisfy (A) and (B). We replace $\mu_{0} \mid Q^{\prime}$ by $\beta_{Q} \cdot \eta \cdot d v_{Q}$, where $\beta_{Q}$ is a constant so that

$$
\mu_{0}\left(Q^{\prime}\right)=\beta_{Q} \int_{E\left(Q^{\prime}\right)} \eta d v_{Q}
$$


The changed measure $\mu_{0}^{\prime}$ satisfies by condition (B)

$$
\int_{E\left(Q^{\prime}\right)} G(z ; \zeta) d v_{0}^{\prime}(\zeta) \leqq \text { Const. }
$$

Since $\mu$ also satisfies (A) it follows from (4.3) that

$$
u^{\prime}(z)=\int G(z ; \zeta) d v_{\mathbf{0}}^{\prime}(\zeta) \leqq \text { Const. on } E .
$$

By the maximum principle

$$
\mu_{\mathbf{0}}^{\prime}(\mathfrak{S})=\mu(\mathfrak{S}) \asymp \int G(z ; \zeta) d v_{\mathbf{0}}^{\prime}(\zeta)=u^{\prime}(i) \leqq \text { Const. } \omega(i) .
$$

This proves the result.

To obtain the Beurling-Nevanlinna projection theorem (in a weak form) we choose for every $y>0$ for which this is possible some $(x, y) \in E$ and define $d \mu_{0}(x, y)=d y$. The corresponding set of $y$ 's is denoted $E^{*}$. Then

$$
\omega(i ; E ; \mathscr{D})>\text { Const. } m E^{*} \text {. }
$$

For Hall's lemma we consider instead $r>0$ so that, for some $\theta, r e^{i \theta} \in E$ and define $d \mu_{0}\left(r e^{i \theta}\right)=d r$. If again the corresponding set of $r$ 's is denoted $E^{*}$, (4.5) holds.

\section{5. $\partial \mathscr{D}$ a curve; $E \subset \partial \mathscr{D}$}

If $\partial \mathscr{D}$ is rectifiable it follows by conformal mapping that $\omega(i ; E, \mathscr{D})$ and length $\ell(E)$ vanish simultaneously. An upper bound

$$
\omega(E)<\frac{\text { Const. }}{\log \frac{1}{\ell(E)}}
$$

can be given with a uniform constant in terms of the general geometry. No lower bound of such nature can exist.

If $\partial \mathscr{D}$ is a general Jordan curve, our knowledge is very unsatisfactory (see [5]). Lavrentiev has shown that it is possible that $\omega(E)>0$ and $\ell(E)=0$. Conversely if $E \subset \cup \mathscr{D}_{v}$ where $\mathscr{D}_{v}$ are discs with radius $r_{v}$ it follows from the Beurling-Nevanlinna projection theorem in $\S 4$ that

$$
\omega\left(E \cap \mathscr{D}_{v}\right) \leqq \text { Const. } r_{v}^{1 / 2} .
$$

Hence if $\sum r_{v}^{1 / 2}$ can be made arbitrarily small, i.e. $\Lambda_{1 / 2}(E)=0$, then $\omega(E)=0$. It can be shown that there exist $\alpha>1 / 2$ so that $\Lambda_{\alpha}(E)=0$ implies $\omega(E)=0$. To prove this for all $\alpha<1$ would be an important progress that would also imply that if $\varphi$ is a conformal mapping of any domain $\mathscr{D}$ onto the unit disc then

$$
\int_{\mathscr{D}} \int\left|\varphi^{\prime}\right|^{p} d x d y<\infty, \quad p<4
$$




\section{E a linear set}

A natural method to deal with harmonic measure is to try to find an iterative scheme of linear inequalities which derive from the Poisson formula for some explicit situation. This seems to have been first used by Benedicks [2]. I shall here describe in detail a situation where the method works.

Let $x_{v},-\infty<v<\infty, x_{0}=0$, be a sequence of points on the real axis, $0<x_{v+1}$ $x_{v}<C$. Assume also $C^{-1} \leqq a_{v} \leqq C$, and that the intervals

$$
\omega_{v}=\left\{x\left|x-x_{v}\right| \leqq a_{v}\right\}
$$

are disjoint. Let $\mathscr{D}$ be the complement of $F=\cup \omega_{v}$. We wish to estimate $\omega\left(z ; \omega_{0} ; \mathscr{D}\right)$ for $z$ real.

Let $I_{v}$ be the complementary intervals of $F$ in natural order. Suppose $m_{v}=$ $\max _{I_{v}} \omega(x)$ is taken at $\xi_{v} \in I_{v}$. Let $K_{v}(x ; t)$ be the Poisson kernel for the plane $\backslash I_{v}$. Then, if $\bar{I}_{v} \cap \bar{\omega}_{0}=\emptyset$,

If we set

$$
\begin{aligned}
m_{v}=\omega\left(\xi_{v}\right) & =\int K_{v}\left(\xi_{v} ; t\right) \omega(t) d t \\
& =\sum_{j \neq v} \int_{I_{j}} K_{v}\left(\xi_{v} ; t\right) \omega(t) d t+\int_{\omega_{0}} K_{v}\left(\xi_{v} ; t\right) d t \\
& \leqq \sum_{j \neq v} m_{j} \int_{I_{j}} K_{v}\left(\xi_{v} ; t\right) d t+O\left(v^{-2}\right) .
\end{aligned}
$$

$$
a_{v j}=\int_{I_{j}} K_{v}\left(\xi_{v} ; t\right) d t, \quad v \neq j
$$

and $a_{v j}=0$ for the two excluded intervals $I_{v}$, the matrix $\left(a_{v j}\right)$ has the following two properties:

where $q$ only depends on $C$;

$$
\sum_{j} a_{v j} \leqq q<1
$$

$$
0<a_{v j} \leqq \frac{K}{(v-j)^{2}}, \quad K=K(C) .
$$

Both inequalities are immediate consequences of the assumptions on the intervals and the explicit form for $K_{v}$.

Lemma. Under the assumptions (6.1) and (6.2)

$$
0 \leqq m_{v} \leqq \sum_{j} a_{v j} m_{j}+\frac{\text { Const. }}{v^{2}}<\text { Const. }
$$

implies

$$
m_{v} \leqq \frac{\text { Const. }}{v^{2}}
$$


Proof. If we write (6.3) in matrix notation it takes the form $(x=m)$

$$
0 \leqq x \leqq A x+c
$$

where $x_{j} \leqq$ Const., $c_{j} \leqq$ Const. $j^{-2}$. Hence

$$
x \leqq A^{n} x+\sum_{0}^{n-1} A^{k} c .
$$

Since $\operatorname{Max}_{j}\left(A^{n} x\right)_{j} \leqq q^{n} \rightarrow 0$ it is sufficient to prove the following: There exists $\varepsilon>0$, only depending on $q$ and $C$, so that if

then

$$
0<x_{j} \leqq \operatorname{Min}\left(\varepsilon, j^{-2}\right)=\alpha_{j}
$$

$$
y_{i}=(A x)_{i} \leqq \frac{1+q}{2} \alpha_{i} .
$$

Let $\mathscr{J}=\varepsilon^{-1 / 2}$. There exists $\delta=\delta(q, C)$ so that if $|i| \leqq(1+\delta) \mathscr{J}$ then (6.4) holds. This is an immediate consequence of (6.1). Take $i=\lambda \mathscr{J}$ with $\lambda \geqq 1+\delta$. Then

The second sum is

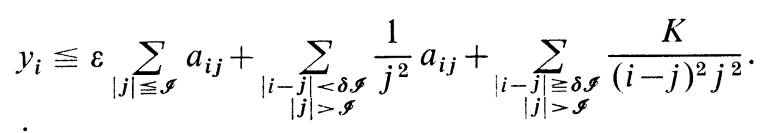

$$
<i^{-2} \frac{2 q+1}{3} \text { for } \delta<\delta(q)
$$

The third sum has an estimate

$$
\text { Const. } i^{-2} \mathscr{I}^{-1}=\text { Const. } i^{-2} \varepsilon^{1 / 2} .
$$

The first sum, finally, is bounded by

$$
\text { Const. } \cdot \varepsilon \int_{(\lambda-1) \mathscr{g}}^{(i+1) \mathscr{I}} \frac{d x}{x^{2}}=\frac{\text { Const. } \varepsilon^{3 / 2}}{i^{2} \varepsilon-1}<\frac{1-q}{6 \cdot i^{2}},
$$

if $\varepsilon$ is sufficiently small, since $i^{2} \varepsilon \geqq 1+\delta$.

In a similar way one can also estimate harmonic measure for compact sets. Let me mention the following results. The proofs will appear elsewhere.

Let $E$ be a compact set so that for every $x_{0} \in E$

$$
m\left(E \cap\left(x_{0}-\delta, x_{0}+\delta\right)\right) \geqq c \delta \text { for all } \delta>0
$$

for some fixed $c>0$. Let dist. $(0, E)=1$, and let $\omega(x)$ denote harmonic measure at 0 for the part of $E$ located in $(0, x)$. Then

(a) $\omega(x)<$ Const. $x^{-\theta}, \theta=\theta(c)>0$.

(b) $\omega$ is absolutely continuous and

$$
\int_{\boldsymbol{E}} \omega^{\prime} \exp \left\{\alpha \omega^{\prime 1 / 2}\right\} d x<\infty . \quad \alpha=\alpha(c)>0 .
$$

A natural conjecture is that $\omega^{\prime} \in L^{p}$ for some $p>1$. 


\title{
References
}

[1] Ahlfors, L.: Untersuchungen zur Theorie der konformen Abbildung und der ganzen Funktionen. - Acta Soc. Sci. Fenn. 1, 1930, 1-40.

[2] Benedicks, M.: Positive harmonic functions vanishing on the boundary of certain domains in $R^{n}$. - Ark. Mat. 18, 1980, 53-72.

[3] Beurling, A.: Etudes sur un problème de majoration. - Thèse, Uppsala, 1933.

[4] Carleman, T.: Sur une inégalité différentielle dans la théorie des fonctions analytiques. - C. R. Acad. Sci. Paris 196, 1933, 995-997.

[5] Carleson, L.: On the distortion of sets on a Jordan curve under conformal mapping. - Duke Math. J. 40, 1973, 547-559.

[6] Hall, T.: Sur la mésure harmonique de certains ensembles. - Ark. Mat. 25, 1937, 1-8.

[7] Nevanlinna, R.: Über eine Minimumaufgabe in der Theorie der konformen Abbildung. Nachr. Ges. Wiss. Göttingen. Fachgruppe I 37, 1933, 103-115.

[8] Nevanlinna, R.: Das harmonische Mass von Punktmengen und seine Anwendung in der Funktionentheorie. - Åttonde skandinaviska matematikerkongressen i Stockholm 14-18 augusti 1934. Comptes rendus du huitième congrès des mathématiciens scandinaves tenu à Stockholm 14-18 août 1934. Håkan Ohlssons boktryckeri, Lund, $116-133$.

[9] Nevanlinna, R.: Eindeutige analytische Funktionen. 2:e Aufl. - Die Grundlehren der mathematischen Wissenschaften 46, Springer-Verlag, Berlin-Göttingen-Heidelberg, 1953.

\author{
Institut Mittag-Leffler \\ Auravägen 17 \\ S-18262 Djursholm \\ Sweden
}

Received 16 June 1981 